\title{
Sur la détermination de la puissance optimum d'aménagement des chutes d'eau
}

Par E. BATICIE, Inginiour an chef des Ponts al Chausse's.

Les débits des cours d'eau susceptibles d'anénagement hydraulique sont en général très variables. Il est commode, pour l'étude des installations, d'utiliser ce qu'on appelle la courbe des débils classés, c'est-à-dire une courbe où sont portés en abscisses les temps pendant lesquels sont assurés les débits représentés par les ordonnées.

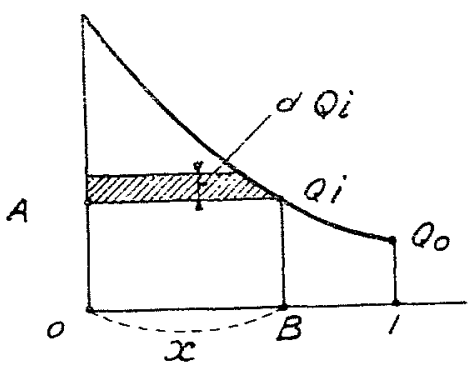

Fïg. 1

Ces courbes sont à ordonnées décroissantes et cette décroissance est d'ailleurs plus ou moins régulière. L'ordonnée maximum $Q_{\mathrm{N}}$, correspondant à une abscisse très petite, est celle de la crue maximum et l'ordonnée minimum correspond à l'étiage absolu $Q_{0}$.

Supposons qu'une installation soit réalisée de manière à capter un débit maximum $Q_{i}, \mathrm{H}$ étant la hauteur de chute (que nous supposons, pratiquement, constante). La puissance installee sera en chevaux :

$$
\mathrm{N}_{\mathrm{i}}=10 \mathrm{HQ} \mathrm{Q}_{\mathrm{i}}
$$

Elle sera réalisée pendant une fraction $x$ d'année.

Le débit moyen correspondant sera représenté par l'aire mixtiligne $O A Q_{i} Q_{0} 1$. Appelons $Q_{m}$ ce débit moyen. Il est lié à $Q_{i}$ par une relation différentielle qui s'établit aisément. Donnons à $Q_{\mathrm{i}}$ un accroissement $d \mathrm{Q}_{\mathrm{i}}$. L'accroissement du débit moyen sera, évidemment, $x d \mathrm{Q}_{\mathrm{i}}$; on a donc :

$$
d \mathrm{Q}_{\mathrm{m}}=x d \mathrm{Q}_{\mathrm{i}} \quad \text { ou } \frac{d \mathrm{Q}_{\mathrm{m}}}{d \mathrm{Q}_{\mathrm{i}}}=x
$$

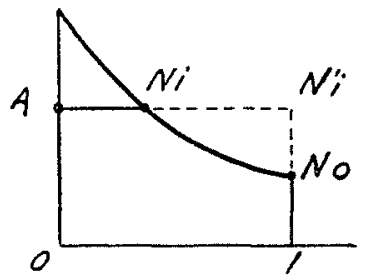

Iig. 2

Si des débits on passe aux puissances, on pourra écrire en appelant $\mathrm{N}_{\mathrm{m}}$ la puissance moyenne, ou, si on veut, l'énergie produite annuellement, en prenant pour unité le chevat-inl, et ell supporsant que la chute est pratiquement constante :

$$
\frac{d \mathrm{~N}_{\mathrm{n}}}{d \mathrm{~N}_{\mathrm{i}}^{-}}=x
$$

Cela posé, le problème de la puissance optimum peut se presenter de deux manières différentes : ou bien on considère l'usine à construire comme isolée et la puissance optimum est la valeur de $N_{i}$ qui rend minimum le prix de revient de $\mathrm{N}_{\mathrm{n}}$; - ou bien l'usine est supposée en parallèle avec une usine thermique ou un rése:ll de distribution qui lui fournit le courant d'appoint et, dans cas, le problème se pose d'une manière un peu différente.

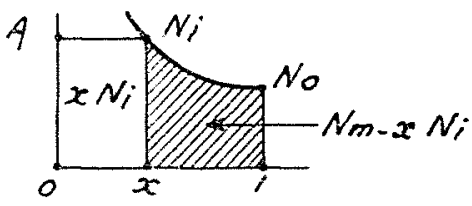

Fig. :3

Soit $\mathrm{AN}_{\mathrm{i}} \mathrm{N}_{0}$ la courbe des puissances classées de l'usinehy dralllique : l'énergie d'appoint a en général pour but de compléter à $N_{i}$ la puissance mise en jeu. Si l'utilisation était intégrale, l'aire $\mathrm{N}_{\mathbf{i}} \mathrm{N}^{\prime} \mathrm{N}_{0}$ représenterait l'énergie d'appoint ; le rectangle $O A N_{i}^{\prime} 1$ représente la capacité totale en énergie de l'ensemble de l'installation.L'énergie écoulée n'est qu'une fraction $\mathrm{KN}_{\mathrm{i}}$ de ce rectangle. La puissance optimum d'installation est celle pour laquelle le quotient des dépenses annu'elles d'ensemble par $\mathrm{KN}_{\mathrm{i}}$ est minimum.

\section{Premier Cas : Usine isolée}

Les dépenses annuelles peuvent loujours être mises grosso modo, sous la forme : $a+b \mathrm{~N}_{\mathrm{i}}$.

Il faut chercher le minimum de $\frac{a+b \mathrm{~N}_{\mathrm{i}}}{\lambda_{\mathrm{m}}}$ : ce minimum a lieu quand on a :

$$
b \mathrm{~N}_{\mathrm{m}}=\left(a+b \mathrm{~N}_{\mathrm{i}}\right) \frac{d \mathrm{~N}_{\mathrm{m}}}{d \mathrm{~N}_{\mathrm{i}}}
$$

Ou en vertu de la relation :

$$
\begin{gathered}
\frac{d \mathrm{~N}_{\mathrm{m}}}{d \mathrm{~N}_{\mathrm{i}}}=x: \\
b \mathrm{~N}_{\mathrm{m}}=\left(a+b \mathrm{~N}_{\mathrm{i}}\right) x
\end{gathered}
$$

Cette relation est équivalente à la suivante :

$$
\frac{b \mathrm{~N}_{\mathrm{i}}}{a}=\frac{\mathrm{N}_{\mathrm{m}}-x \mathrm{~N}_{\mathrm{i}}}{x \mathrm{~N}_{\mathrm{i}}}
$$


Or, $\mathrm{N}_{\mathrm{m}}-x \mathrm{~N}_{\mathrm{i}}$ est représenté par l'aire $x \mathrm{NN}_{\mathrm{i}_{0}} 1$. C'est l'énergie fournie quand le débit est inférieur au débit d'installation ; on peut donc dire qus la puissance optimum d'une usine isolée est celle pour laquelle le rapport des charges annuelles constantes aux charges variables avec la puissance, est égal au rapport de la capacité en énergie de l'installation pendant la période où le débit est supérieur ou égal au débit d'installation à la capacité en énergie pendant la période oil le débit est inférieur au débit d'installation.

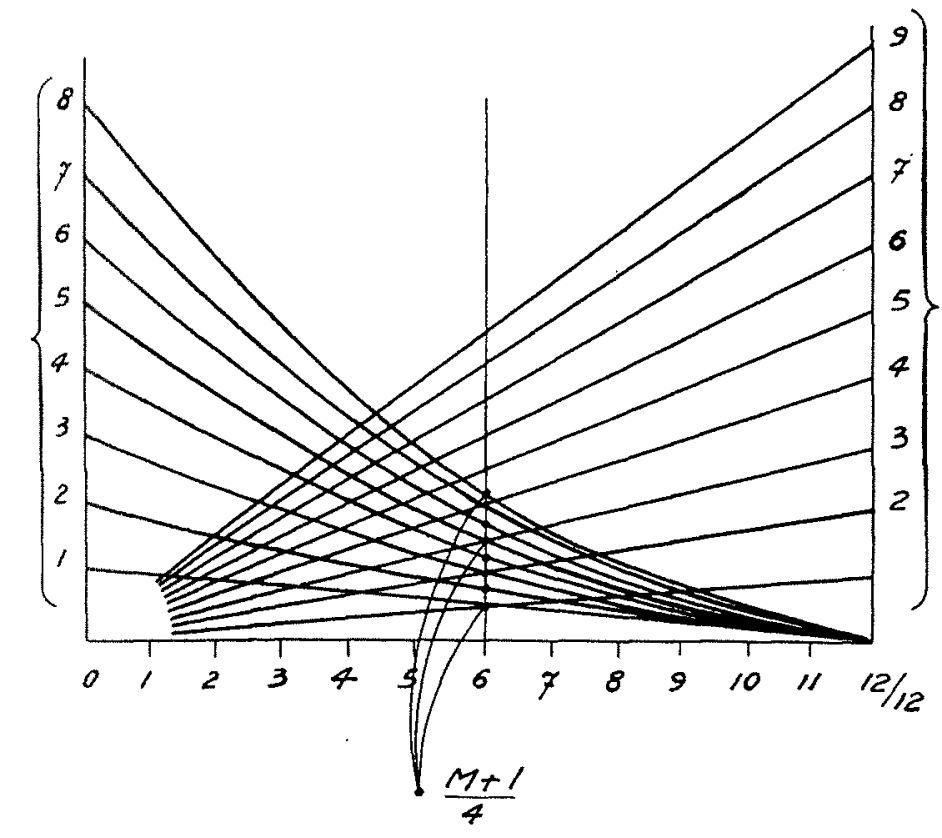

Fig. 4

Deuxieme Cas : Usine avec Énergie d'appoint

Les dépenses annuelles de l'usine proprement dite peuvent encore être mises sous la forme $a+b \mathrm{~N}_{\mathbf{i}}$.

Quant aux dépenses annuelles afférentes à l'énergie d'appoint, elles peuvent bien encore se mettre sous la forme binôme, mais les deux termes seront l'un proportionnel à la puissance installée $\left(N_{1}-N_{0}\right)$, et l'autre proportionnel à l'énergie d'appoint consommée $\mathrm{K}\left(\mathrm{N}_{\mathrm{i}}-\mathrm{N}_{\mathrm{m}}\right)$.

C'est sous cette forme que se présentent en général les tarifs des entreprises de distribution et c'est à cette forme que peuvent se ramener les dépenses d'une usine thermique d'appoint.

Les dépenses annuelles totales seront donc représentées par l'expression :

$$
a+b \mathrm{~N}_{\mathbf{i}}+a\left(\mathrm{~N}_{\mathbf{i}}-\mathrm{N}_{0}\right)+\beta \mathrm{K}\left(\mathrm{N}_{\mathbf{i}}-\mathrm{N}_{\mathrm{m}}\right)
$$

Le prix de revient à rendre minimum est :

$$
p=\frac{a+b \mathrm{~N}_{\mathrm{i}}+x\left(\mathrm{~N}_{\mathrm{i}}-\mathrm{N}_{0}\right)+\beta \mathrm{K}\left(\mathrm{N}_{\mathrm{i}}-\mathrm{N}_{\mathrm{m}}\right)}{\mathrm{KN}_{\mathrm{i}}}
$$

Ou encore :

$$
p=\frac{a-\alpha \mathrm{N}_{0}-\beta \mathrm{KN}_{\mathrm{m}}}{\mathrm{KN}_{\mathrm{i}}}+\frac{b+\alpha}{\mathrm{K}}+\beta
$$

Lee minimum a lieu en même temps que celui de

$$
\frac{a-\alpha N_{0}-\beta \mathrm{KN}_{\mathrm{n}}}{\mathrm{KN}_{\mathrm{i}}}
$$

En tenant compte de ce que $\frac{d \mathrm{~N}_{\mathrm{m}}}{d \mathrm{~N}_{\mathrm{i}}}=x$ on trouve en dérivant :

$$
a-\alpha \mathrm{N}_{0}-\beta \mathrm{KN}_{\mathrm{m}}=\beta \mathrm{K} x \mathrm{~N}_{\mathrm{i}}
$$

ou :

$$
a-\alpha \mathrm{N}_{0}=\beta \mathrm{K}\left(\mathrm{N}_{\mathrm{m}}-x \mathrm{~N}_{\mathrm{i}}\right)
$$

Cette relation est équivalente à la suivante :

$$
\alpha \mathrm{N}_{\mathrm{i}}+\beta \mathrm{K}\left(\mathrm{N}_{\mathrm{m}}-x \mathrm{~N}_{\mathrm{i}}\right)=a+\alpha\left(\mathrm{N}_{\mathrm{i}}-\mathrm{N}_{0}\right)
$$

On retrouve la quantité $\mathrm{N}_{m}-x \mathrm{~N}_{i}$ qui représente l'énergie que l'usine hydraulique est capable de produire quand le débit est au-dessous du débit d'installation, c'est-à-dire quand on fait appel à l'énergie d'appoint.

$\approx \mathrm{N} i+\beta \mathrm{K}\left(\mathrm{N}_{\mathrm{m}}-x \mathrm{~N}_{\mathrm{i}}\right)$ est le prix de l'énergie hydraulique $\underline{a}$ correspondante supposé évalué au tarif de l'énergie d'appoint, puisque la puissance installée correspondante est $\mathrm{N}_{\mathrm{i}}$. D'autre part, $a$ et $\alpha\left(\mathrm{N}_{\mathbf{i}}-\mathrm{N}_{0}\right)$ représentant les charges constantes de chacune des sources d'énergie, les mots " charges constantes" désignant les dépenses indépendantes de $\mathrm{N}_{\mathrm{i}}$ pour l'usine hydraulique et celles indépendantes de $N_{m}$ pour l'énergie d'appoint.

Nous pouvons donc formuler la conclusion suivante :

La puissance optimum d'une usine hydraulique adjointe à une usine thermique ou à une distribution publique destinée à fournir le courant d'appoint est celle pour laquelle le prix de l'énergie hydraulique fournie quand on fait appel à l'énergie d'appoint, supposé évalué au tarif de l'énergie d'appoint, est égal à la somme des charges constantes de chacune des deux sources d'énergie.

Remarque. - Les règles que nous venons d'énoncer présentent un certain intérêt pour des vérifications a posteriori. Mais elles perdent leur avantage comme méthodes de recherche.

Pratiquement on construira les courbes $\mathrm{N}_{\mathrm{m}}$ et $x \mathrm{~N}_{\mathrm{i}}$.

D'où $\mathrm{N}_{\mathrm{m}}-x \mathrm{~N}_{\mathrm{i}}$. Le point optimum s'obtient par l'intersection de cette courbe $\mathrm{N}_{\mathrm{m}}-x \mathrm{~N}_{\mathrm{i}}$ :

10 Avec la droite $\frac{a x}{b}$, dans le cas de l'usine isolée;

$2^{\circ}$ Avec la droite $\frac{a-x N_{0}}{\hat{k} \mathrm{~K}}$ dans le cas de l'usine adjointe à une source d'énergie d'appoint.

\section{$\star^{\star} \star$}

Application. - Nous allons montrer comment on peut pratiquement, moyennant certaines hypothèses simplificatrices sur les courbes de débits, utiliser cette méthode générale à la fixation rapide du débit optimum d'installation d'une chute d'eau.

Nous supposons que, dans la partie correspondant à des débits pratiquement utilisables, les courbes des débits classés sont des droites. Nous pouvons alors définir ces courbes par deux points seulement : le débit d'étiage pour $x=1$, pour lequel on a $N_{i}=$ $\mathrm{N}_{0}$, et le débit caractéristique moyen correspondant $\grave{\mathrm{a}} x=\frac{1}{2}$ et pour lequel on a $N_{f}=\mu . N_{0}$. Le coefficient ; pourra servir à caractériser le régime et nous l'appelons Coefficient d'irrégularité. 
Dans ces conditions on voit aisément qu'on aura :

$$
\begin{aligned}
& \frac{\mathrm{N}_{\mathrm{i}}}{\mathrm{N}_{0}^{-}}=2 \mu-1-2(\mu-1) x \\
& \frac{N_{\text {m }}}{N_{0}}=\mu-(\mu-1) x^{2} \\
& \frac{1}{N_{0}}\left(\mathrm{~N}_{\mathrm{m}}-x \mathrm{~N}_{\mathrm{j}}\right)=(\mu-1) x^{2}-(2 \mathrm{\mu}-1) x+\mu=y
\end{aligned}
$$

Ces dernières courbes passent toutes par $y=0$ et $x=\mathrm{I}$. D'autre part l'ordonnée à l'origine est $\mu$. . Le sommet a pour coordonnées

$$
x=\frac{2 \mu-1}{2(\mu-1)} \quad(>1) \quad \text { et } y=-\frac{1}{4} \frac{1}{\mu-1} .
$$

L'ordonnée pour $x=\frac{1}{2}$ est $y=\frac{\mu+1}{4}$.

Le point correspondant à la puissance optimum s'obtient dans le cas de l'usine isolée par l'intersection de la courbe $y N_{0}$ avec $y=\frac{a x}{b}$.

Mais $a$ est évidemment proportionnel à $\mathrm{N}_{0}$ : nous posons $a=a^{\prime} \mathrm{N}_{0}$ de sorte que le point de puissance optimum est à l'intersection de $y=\frac{a^{\prime} x}{b}$ et de $y=(u-1) x^{2}-(2 \mu-\mathrm{I}) x+\mu$.

$\frac{a^{\prime}}{b}$ peut, grosso modo, être considéré comme le rapport du prix par cheval installé de la partie électro-mécanique de l'installation au prix par cheval d'étiage de la partie hydraulique.

Par exemple soit une usine de 10.000 chevaux installés, ayant coûté 10.000 .000 de fr., la puissance d'étiage étant de 4.000 chevaux. Supposons que la partie électro-mécanique coûte $400 \mathrm{fr}$. par cheval, il reste pour la partie hydraulique 6.000 .000 ; d'où par cheval d'étiage : $\frac{6.000 .000}{4.000}=1.500 \mathrm{fr}$; on aura : $\frac{a^{\prime}}{b}=3,7$.

Soit une autre usine de 8.000 chevaux installés ayant coûté 6.000 .000 de fr., la puissance d'étiage n'est que de 2.000 chevaux. La partie électro-mécanique est moins chère en raison de la chute plus forte (nombre de tours plus grand), soit 300 fr. La partie hydraulique ressort à $6.000 .000-2.400 .000=3.600 .000$ francs. D'où par cheval d'étiage ':

$$
\frac{3.600 .000}{2.000}=1.800 \text { fr. ; par suite } \frac{a^{\prime}}{b}=6 .
$$

Troisième exemple; Usine de 6.900 chevaux ayant coûté 5.000 .000 de francs.

Etiage 1.000 chevaux ; partie électro-mécanique : $300 \mathrm{fr}$. par cheval; partie hydraulique $: 5.000000-2.070 .000=2.930 .000 \mathrm{fr}$.
D'où par cheval d'étiage : 2.930 fr. D'où $\frac{a^{\prime}}{b}=9, \mathrm{~b}$.

Ces exemples qui n'ont pas été pris tout à fait arbitrairement, montrent qu'il est toujours facile d'évaluer avec un avant projet même sommaire, l'ordre de grandeur du rapport $\frac{a^{\prime}}{b}$ qui carac. térise l'importance des installations de génie civil par rapport aux installations mécaniques. Dune manière générale, nous estimons que, pour les bonnes chutes alpestres, sans barrage réservoir, ce coefficient est de l'ordre de 5 . Le coefficient $\mu$ descend rarement au-dessous de 3 . Le graphique ci-dessous donne les courbes

$$
y=\frac{\mathrm{N}}{\mathrm{N}_{\theta}}\left(\mathrm{N}_{\mathrm{m}}-x \mathrm{~N}_{\mathrm{i}}\right) \text { et } y=\frac{a^{\prime}}{b} x .
$$

Les abcisses sont divisées en $1 / 12$, c'est-à-dire en mois. Il permet de déterminer immédiatement par la connaissance des deux coefficients $\frac{a^{\prime}}{b}$ et $\mu$. le débit optimum de l'installation. Par exemple on voit que si $\frac{a^{\prime}}{b}$ a la valeur moyenne 5 et si p. est égal à 3 il faudra équiper la chute pour le débit de 4 mois environ.

Dans le cas de l'usine adjointe à un secteur, on prendra l'inter. section des courbes $y(\mu)$ avec les droites $\frac{a^{\prime}-x}{\beta \mathrm{K}}$. En général les deux parties du tarif binôme sont égales pour la valeur moyenne du coefficient d'utilisation. Donc $\alpha=\beta \mathrm{K}$.

Supposons cette valeur égale à $200 \mathrm{fr}$., ce qui est admissible

Considérons une des usines mentionnées ci-dessus, celle pour laquelle $\frac{a^{\prime}}{b}=6: a^{\prime}$ représente dans notre approximation le coult annuel de la partie hydraulique par cheval d'étiage; admettons le taux moyen de $12 \%$ (amortissement, intérêt, frais constants d'exploitation), on aura : $a^{\prime}=1.800 \times 0,12=220$, et par suite $\frac{a^{\prime}-x}{\mathrm{~K} K}=0,10$. La puissance optimum serait dans cette hypo thèse très voisine de la puissance d'étiage.

Dans le troisième exemple on aurait $a^{\prime}=350 \mathrm{fr}$., alors $\frac{a^{\prime}-\alpha}{\beta \mathrm{K}}=\frac{150}{200}=0,75$. Pour $\mu=3$, la puissance optimum est celle qui correspond au débit de 7 mois $1 / 2$ environ.

Examinons enfin le cas où l'energie d'appoint serait fournip par un moteur Diesel. Admettons un prix d'installation de 900ft. par cheval avec du combustible à $300 \mathrm{fr}$. la tonne ; on aura $\alpha=100 \mathrm{fr}$. (intérêt, amortissement, surveillance et entretien); la consommation sera d'environ 2,65 $\mathrm{T}$ par cheval-an ; d'où aveé l'huile : $\beta=800$; pour $\mathrm{K}=0,25$ on aura $\beta \mathrm{K}=200$. Dans ces conditions on trouverait pour la troisième usine: $\frac{a^{\prime}-\alpha}{\beta \mathrm{K}}=\frac{250}{20}=1,25$.

L'équipement optimum pour un cours d'eau dont le coefif cient d'irrégularité serait de 3 , serait celui du débit de 5 mois. 